081 SPIKES IN EXTERNAL TRAINING LOAD ARE ASSOCIATED WITH SHOULDER INJURIES IN COMPETITIVE ADOLESCENT TENNIS PLAYERS - THE SMASH COHORT STUDY

\begin{abstract}
1,2,3Fredrik Johansson, ${ }^{4}$ Ann Cools, ${ }^{5} J a i m e$ Fernandez-Fernandez, ${ }^{6,7}$ Tim Gabbett,
1,2,3Eva Skillgate. 'Musculoskeletal and Sports Injury Epidemiology Center, Sophiahemmet University, Stockholm, Sweden; ${ }^{2}$ Intervention and Implementation research for worker health, Institute of Environmental Medicine, Karolinska Institutet, Stockholm, Sweden; ${ }^{3}$ Naprapathögskolan - Scandinavian College of Naprapathic Manual Medicine, Stockholm, Sweden; ${ }^{4}$ Department of Rehabilitation Sciences and Physiotherapy, Faculty of Medicine and Health Sciences, Ghent University Hospital, Ghent, Belgium; ' Department of Physical Activity and Sports Sciences, Universidad de León, León, Spain; ${ }^{6}$ Gabbett Performance Solutions, Brisbane, Australia; ${ }^{7}$ Centre for Health Research, University of Southern Queensland, Ipswich, Australia
\end{abstract}

\subsection{6/bjsports-2021-IOC.77}

Background Few studies have examined the association between the acute:chronic workload ratio (ACWR) and complaints/injuries in young tennis players. Primary aims: to investigate if accumulated external workload 'spikes' in ACWR of tennis training, match-play and fitness training, and if high or low workload/age ratio, were associated with the rate of shoulder complaints/injuries in competitive adolescent tennis players. Additional aims: to report the incidence of complaints/injuries stratified by sex and level of play, and describe shoulder injury characteristics.

Hypothesis Rapid increases in external workload are associated with the incidence of shoulder complaints and injuries.

Study design Cohort study.

Level of evidence 3

Methods At baseline 301 adolescent competitive tennis players, 13-19 years, were screened and followed weekly for 52 weeks with questionnaires, in years 2018-2019.

Information about time varying accumulated external workload spikes (uncoupled ACWR >1.3), and workload/age ratio, in 252 uninjured players were used in Cox regression analyses with the outcomes shoulder complaints $(\geq 20)$ and injuries $(\geq 40)$ (Oslo Sports Trauma Research Center Overuse Injury Questionnaire).

Results For each additional workload spike in tennis training/ match-play the Hazard Rate Ratio (HRR) was 1.26 (95\% CI: 1.13 - 1.40) for a shoulder complaint, and 1.26 (95\% CI: 1.15 - 1.39) for a shoulder injury. The HRR for fitness training was 1.11 (95\% CI: $1.02-1.20$ ) for a shoulder complaint, and 1.18 (95\% CI: 1.09 - 1.27) for a shoulder injury. Workload/age ratio was not associated to the rate of shoulder complaints or injuries. Conclusion Accumulated external workload spikes of tennis training, match-play and/or fitness training are associated with a higher rate of shoulder complaints and shoulder injuries in competitive adolescent tennis players.

Clinical relevance Consistency in training load on a weekly basis is most likely more beneficial for adolescent tennis players regarding shoulder complaints/injuries than a training schedule comprising rapid increases (i.e. spikes) in workload.

\section{TRAINING LOAD AND OTHER RISK FACTORS FOR SOFT TISSUE INJURY RISK IN PROFESSIONAL RUGBY UNION: A 13 TEAM, 2-SEASON STUDY OF 383 INJURIES}

\footnotetext{
${ }^{1}$ Stephen West, ${ }^{1}$ Sean Williams, ${ }^{1}$ Dario Cazzola, ${ }^{2}$ Matthew Cross, ${ }^{3}$ Simon Kemp, ${ }^{1,3}$ Keith Stokes. 'University of Bath, Bath, UK; ${ }^{2}$ Premier Rugby Limited, London, UK; ${ }^{3}$ Rugby Football Union, London, UK
}

10.1136/bjsports-2021-IOC.78
Background The risk of injury in professional rugby union is high compared with other sports. Modifiable risk factors such as training load provide an attractive target for risk management.

Objective To explore the influence of training load and other known risk factors on soft tissue injury risk.

Design Observational cohort.

Setting Thirteen professional (English Premiership) rugby clubs over two seasons.

Patients (or Participants) During two seasons, 433 and 569 players were recruited, resulting in 1002 player-seasons from 696 unique players.

Interventions (or Assessment of Risk Factors) Training load metrics (session Rating of Perceived Exertion), previous injury, previous concussion, match minutes, age and position.

Main Outcome Measurements Soft tissue injury hazard (daily risk).

Results Age and position were unclear risk factors for injury (p-values: $0.20-0.49$ for age and $0.40-0.97$ for position). Players without a previous injury were at the lowest risk of subsequent injury, while the greater the number of previous injuries, the greater the risk for subsequent injury. Playing 'moderate-high' and 'high' match minutes was associated with a lower risk of injury than players with 'low' match minutes. Unexpectedly, having a previous concussion was associated with a lower risk of injury than players without a previous concussion. For training load measures, 3-day acute load showed no clear relationship with injury risk, while moderatehigh 14-day chronic loads (248-337 daily AU) demonstrated a 'likely' harmful effect on injury risk (Relative Risk: 1.4, 95\% CIs:1.1-1.8).

Conclusions Clear associations between injury risk and chronic training load measures were identified, as well as associations with previous injury, previous concussion and match minutes. These findings support the need for careful individualised load management and athlete profiling to include other risk factors (e.g., previous injury, previous concussion and match minutes). This should be undertaken to inform risk management decisions in athlete training programmes.

\section{WORKLOAD WEIGHTED FOR TISSUE DAMAGE RESULTS IN HIGHER ACUTE:CHRONIC WORKLOAD RATIO FOR INJURED VS. UNINJURED ATHLETES}

${ }^{1}$ Lauren Benson, ${ }^{1,2}$ Oluwatoyosi Owoeye, ${ }^{1}$ Carlyn Stilling, ${ }^{3,4} \mathrm{~W}$ Brent Edwards, ${ }^{1,4,5,6,7}$ Carolyn Emery. ${ }^{1}$ Sport Injury Prevention Research Centre, Faculty of Kinesiology, University of Calgary, Calgary, Canada; ${ }^{2}$ Department of Physical Therapy and Athletic Training, Doisy College of Health Sciences, Saint Louis University, St. Louis, USA; ${ }^{3}$ Faculty of Kinesiology, University of Calgary, Calgary, Canada; ${ }^{4}$ McCaig Bone and Joint Institute, Cumming School of Medicine, University of Calgary, Calgary, Canada; ${ }^{5}$ Alberta Children's Hospital Research Institute, University of Calgary, Calgary, Canada; ${ }^{6}$ Department of Community Health Sciences, Cumming School of Medicine, University of Calgary, Calgary, Canada; 'Department of Pediatrics, Cumming School of Medicine, University of Calgary, Calgary, Canada

\subsection{6/bjsports-2021-IOC.79}

Background Risk of overuse injury is often monitored through the acute:chronic workload ratio (ACWR), where workload is the quantity or magnitude of loading cycles. However, weighting the loading magnitude by raising it to a power equal to the slope of the stress-life curve for the tissue may give a better estimate of the damage accumulated due to workload. 